\title{
Onset of Classical Behaviour After a Phase Transition
}

\author{
R. J. Rivers ${ }^{1}$ and F. C. Lombardo ${ }^{2}$ \\ ${ }^{1}$ Theoretical Physics Group; Blackett Laboratory, Imperial College, London SW7 2BZ \\ ${ }^{2}$ Departamento de Física, Facultad de Ciencias Exactas y Naturales, Universidad de Buenos Aires \\ Ciudad Universitaria, Pabellón I, 1428 Buenos Aires, Argentina
}

Received on 20 January, 2005

\begin{abstract}
We analyze the onset of classical behaviour in a scalar field after a continuous phase transition, in which the system-field, the long wavelength order parameter of the model, interacts with an environment of its own shortwavelength modes. We compute the decoherence time for the system-field modes from the master equation and compare it with the other time scales of the model. Within our approximations the decoherence time is in general the smallest dynamical time scale. Demanding diagonalisation of the decoherence functional produces identical results. The inclusion of other environmental fields makes diagonalisation occur even earlier.
\end{abstract}

\section{Introduction}

The standard big bang cosmological model of the early universe assumes a period of rapid cooling, giving a strong likelihood of phase transitions, at the grand unified and electroweak scales [1] in particular. What interests us in this talk is the way in which phase transitions naturally take us from a quantum to classical description of the universe.

That (continuous) transitions should move us rapidly to classical behaviour is not surprising. Classical behaviour has two attributes:

- Classical correlations: By this is meant that the Wigner function(al) $W[\pi, \phi]$ peaks on classical phasespace trajectories, with a probabilistic interpretation.

- Diagonalisation: By this is meant that the density matrix $\rho(t)$ should become (approximately) diagonal, in this case in a field basis. Alternatively, we can demand diagonalisation of the decoherence functional. In either case a probabilistic description (no quantum interference) is obtained.

From the papers of Guth and Pi [2] onwards, it has been appreciated that unstable modes lead to correlations through squeezing. On the other hand, we understand diagonalisation to be an almost inevitable consequence of tracing over the 'environment' of the 'system' modes.

Continuous transitions supply both ingredients. Firstly, the field ordering after such a transition is due to the growth in amplitude of unstable long-wavelength modes, which arise automatically from unstable maxima in the potential. Secondly, the stable short-wavelength modes of the field, together with all the other fields with which it interacts, form an environment whose coarse-graining enforces diagonalisation and makes the long-wavelength modes decohere.

While there are few doubts about the classical outcome, to quantify these general observations is difficult because, with fields, we are dealing with infinite degree of freedom systems. One of us (F.L) has shown elsewhere [3] how classical correlations arise in quantum mechanical systems that mimic the field theory that we shall consider here, and we refer the reader to that paper for the role that classical correlations play. Our concern in this talk is, rather, with diagonalisation, equally necessary for the onset of classical behaviour. This is determined both through the master equation for the evolution of the density matrix and the decoherence functional, whose role is to describe consistent histories.

This talk builds upon earlier work by us $[4,5,6]$, and we refer the reader to it for much of the technical details. We restrict ourselves to flat space-time. The extension to nontrivial metrics is straightforward in principle. See the talk of Lombardo in these same proceedings [7], which complements this.

\section{Basic Ideas}

The evolution of a quantum field as it falls out of equilibrium at a transition is determined in large part by its behaviour at early times, before interactions have time to take effect. To be concrete, consider a real scalar field $\phi(x)$, described by a $Z_{2}$-symmetry breaking action $\left(\mu^{2}>0\right)$

$$
S[\phi]=\int d^{4} x\left\{\frac{1}{2} \partial_{\mu} \phi \partial^{\mu} \phi+\frac{1}{2} \mu^{2} \phi^{2}-\frac{\lambda}{4 !} \phi^{4}\right\} .
$$

with symmetry breaking scale $\eta^{2}=6 \mu^{2} / \lambda$. On heating, this shows a continuous transition, with critical temperature $T_{\mathrm{c}}^{2}=2 \eta^{2}$. If, by virtue of the expansion of the universe the system is very rapidly cooled (quenched) from $T>T_{\mathrm{c}}$ to $T<T_{\mathrm{c}}$, the initial stages of the transition can be described by a free field theory with inverted mass $-\mu^{2}<0$. The state of the field is initially concentrated on the local maximum of the potential, and spreads out with time. This description is 
valid for short times, until the field wave functional explores the ground states of the potential.

The $\phi$-field ordering after the transition is due to the growth in amplitude of its unstable long-wavelength modes, which we term $\phi_{<}(x)$. For an instantaneous quench these have wave-number $k<\mu$ for all time. Although the situation is more complicated for slower quenches, until the transition is complete there are always unstable modes. As a complement to these, we anticipate that the stable shortwavelength modes of the field $\phi_{>}(x)$, where

$$
\phi(x)=\phi_{<}(x)+\phi_{>}(x),
$$

will form an environment whose coarse-graining makes the long-wavelength modes decohere [8]. In practice, the boundary between stable and unstable is not crucially important, provided there is time enough for the power in the field fluctuations to be firmly in the long-wavelength modes. This requires weak coupling $\lambda \ll 1$. Of course, all the other fields with which $\phi$ interacts will contribute to its decoherence, but for the moment we ignore such fields (before returning to them in the last section).

After splitting, the action (1) can be written as

$$
S[\phi]=S\left[\phi_{<}\right]+S\left[\phi_{>}\right]+S_{\mathrm{int}}\left[\phi_{<}, \phi_{>}\right],
$$

where the interaction term is dominated $[4,6]$ by its biquadratic term

$$
S_{\mathrm{int}}\left[\phi_{<,}, \phi_{>}\right] \approx-\frac{1}{6} \lambda \int d^{4} x \phi_{<}^{2}(x) \phi_{>}^{2}(x) .
$$

The total density matrix (for the system and bath fields) is defined by

$$
\rho_{\mathrm{r}}\left[\phi^{+}, \phi^{-}, t\right]=\rho\left[\phi_{<}^{+}, \phi_{>}^{+}, \phi_{<}^{-}, \phi_{>}^{-}, t\right]=\left\langle\phi_{<}^{+} \phi_{>}^{+}|\hat{\rho}| \phi_{<}^{-} \phi_{>}^{-}\right\rangle,
$$

and we assume that, initially, the thermal system and its environment are not correlated.

On tracing out the short-wavelength modes, the reduced density matrix

$$
\rho_{\mathrm{r}}\left[\phi_{<}^{+}, \phi_{<}^{-}, t\right]=\int \mathcal{D} \phi_{>} \rho\left[\phi_{<}^{+}, \phi_{>}, \phi_{<}^{-}, \phi_{>}, t\right],
$$

whose diagonalisation determines the onset of classical behaviour, evolves as

$$
\rho_{\mathrm{r}}[t]=\int d \phi_{<i}^{+} \int d \phi_{<i}^{-} J_{\mathrm{r}}\left[t, t_{i}\right] \rho_{\mathrm{r}}\left[t_{i}\right],
$$

where $J_{\mathrm{r}}\left[t, t_{i}\right]$ is the evolution operator

$$
J_{\mathrm{r}}\left[t, t_{i}\right]=\int_{\phi_{<i}^{+}}^{\phi_{<f}} \mathcal{D} \phi_{<} \int_{\phi_{<i}}^{\phi_{<f}} \mathcal{D} \phi_{<} \exp \left\{i S_{C G}\left[\phi_{<}^{+}, \phi_{<}^{-}\right]\right\} .
$$

$S_{C G}\left[\phi_{<}^{+}, \phi_{<}^{-}\right]$is the coarse-grained effective action, of the closed time-path form

$$
S_{C G}\left[\phi_{<}^{+}, \phi_{<}^{-}\right]=S\left[\phi_{<}^{+}\right]-S\left[\phi_{<}^{-}\right]+\delta S\left[\phi_{<}^{+}, \phi_{<}^{-}\right] .
$$

All the information about the effect of the environment is encoded in $\delta S\left[\phi_{<}^{+}, \phi_{<}^{-}\right]$through the influence functional (or Feynman-Vernon functional [9])

$$
F\left[\phi_{<}^{+}, \phi_{<}^{-}\right]=\exp \left\{i \delta S\left[\phi_{<}^{+}, \phi_{<}^{-}\right]\right\},
$$

giving $\delta S$ a well defined diagrammatic expansion.

\section{The Master Equation}

To see how the diagonalisation of $\rho_{\mathrm{r}}$ occurs, we construct the master equation, which casts its evolution in differential form. As a first approximation, we make a saddle-point approximation for $J_{r}$ in (8),

$$
J_{\mathrm{r}}\left[\phi_{\mathrm{f}}^{+}, \phi_{\mathrm{f}}^{-}, t_{f} \mid \phi_{\mathrm{i}}^{+}, \phi_{\mathrm{i}}^{-}, t_{i}\right] \approx \exp \left(i S_{C G}\left[\phi_{\mathrm{cl}}^{+}, \phi_{\mathrm{cl}}^{-}\right]\right),
$$

In (11) $\phi_{\mathrm{cl}}^{ \pm}$is the solution to the equation of motion

$$
\left.\frac{\delta R e S_{C G}}{\delta \phi^{+}}\right|_{\phi^{+}=\phi^{-}}=0
$$

with boundary conditions $\phi_{\mathrm{cl}}^{ \pm}\left(t_{0}\right)=\phi_{\mathrm{i}}^{ \pm}$and $\phi_{\mathrm{cl}}^{ \pm}(t)=\phi_{\mathrm{f}}^{ \pm}$.

It is very difficult to solve this equation analytically. We exploit the fact that, after the transition, the field cannot be homogeneous in one of its groundstates $\phi=\eta$ or $\phi=-\eta$ because of causality [10]. As a result there is an effective 'domain' structure in which the domain boundaries are 'walls' across which $\phi$ flips from one groundstate to the other. Further, these domains have a characteristic size $\xi$, where $\xi^{-1}=\pi k_{0}$ labels the dominant momentum in the power of the $\phi$-field fluctuations as the unstable longwavelength modes grow exponentially. For simplicity, we adopt a 'minisuperspace' approximation, in which we assume regular domains, enabling $\phi_{\mathrm{cl}}(\vec{x}, s)$ to be written as

$$
\phi_{\mathrm{cl}}(\vec{x}, s)=f(s, t) \Phi(x) \Phi(y) \Phi(z),
$$

where $\Phi(0)=\Phi(\xi)=0$, and

$$
\Phi(x+\xi)=-\Phi(x) .
$$

$f(s, t)$ satisfies $f(0, t)=\phi_{\mathrm{i}}$ and $f(t, t)=\phi_{\mathrm{f}}$. We write it as

$$
f(s, t)=\phi_{\mathrm{i}} u_{1}(s, t)+\phi_{\mathrm{f}} u_{2}(s, t) .
$$

In [6] we made the simplest choice for $\Phi(x)$,

$$
\Phi(x)=\sin k_{0} x
$$

Extensions to include more Fourier modes are straightforward in principle, but our work in [6] was sufficient to show that the results only depend weakly on the details of the domain function $\Phi(x)$ for few Fourier modes. In the light of the more qualitative comments made here, we refer the reader again to [6] for details. On the other hand, the $u_{i}(s, t)$ are solutions of the mode equation for wavenumber $k_{0}$ during the quench, with boundary conditions $u_{1}(0, t)=1$, $u_{1}(t, t)=0$ and $u_{2}(0, t)=0, u_{2}(t, t)=1$.

In order to obtain the master equation we must compute the final time derivative of the propagator $J_{\mathrm{r}}$. After that, all 
the dependence on the initial field configurations $\phi_{\mathrm{i}}^{ \pm}$(coming from the classical solutions $\phi_{\mathrm{cl}}^{ \pm}$) must be eliminated. Assuming that the unstable growth has implemented diagonalisation before back-reaction is important, $J_{r}$ can be determined, approximately, from the free propagators as

$$
J_{0}\left[t, t_{i}\right]=\int_{\phi_{<i}^{+}}^{\phi_{<f}} \mathcal{D} \phi_{<} \int_{\phi_{<i}}^{\phi_{<f}} \mathcal{D} \phi_{<} \exp \left\{i\left[S_{0}\left(\phi^{+}\right)-S_{0}\left(\phi^{-}\right)\right]\right\}
$$

where $S_{0}$ is the free-field action. This satisfies the general identities [8]

$\phi_{\mathrm{cl}}^{ \pm}(s) J_{0}=\left[\phi_{\mathrm{f}}^{ \pm}\left[u_{2}(s, t)-\frac{\dot{u}_{2}(t, t)}{\dot{u}_{1}(t, t)} u_{1}(s, t)\right] \mp i \frac{u_{1}(s, t)}{\dot{u}_{1}(t, t)} \partial_{\phi_{<\mathrm{f}}^{ \pm}}\right] J_{0}$

which allow us to remove the initial field configurations $\phi_{\mathrm{i}}^{ \pm}$, and obtain the master equation.

Even with these simplifications the full equation is very complicated, but it is sufficient to calculate the correction to the usual unitary evolution coming from the noise (diffusion) kernels (to be defined later). The result reads

$$
i \dot{\rho}_{\mathrm{r}}=\left\langle\phi_{<\mathrm{f}}^{+}\left|\left[H, \rho_{\mathrm{r}}\right]\right| \phi_{<\mathrm{f}}^{-}\right\rangle-i V \Delta^{2} D\left(\omega_{0}, t\right) \rho_{\mathrm{r}}+\ldots
$$

where $D$ is the diffusion coefficient and

$$
\Delta=\left(\phi_{\mathrm{f}}^{+2}-\phi_{\mathrm{f}}^{-2}\right) / 2
$$

for the final field configurations (henceforth we drop the suffix). The ellipsis denotes other terms coming from the time derivative that do not contribute to the diffusive effects. $V$ is understood as the minimal volume inside which there are no coherent superpositions of macroscopically distinguishable states for the field.

The effect of the diffusion coefficient on the decoherence process can be seen by considering the following approximate solution to the master equation:

$\rho_{\mathrm{r}}\left[\phi_{<}^{+}, \phi_{<}^{-} ; t\right] \approx \rho_{\mathrm{r}}^{\mathrm{u}}\left[\phi_{<}^{+}, \phi_{<}^{-} ; t\right] \exp \left[-V \Delta^{2} \int_{0}^{t} d s D\left(k_{0}, s\right)\right]$,

where $\rho_{\mathrm{r}}^{\mathrm{u}}$ is the solution of the unitary part of the master equation (i.e. without environment). The system will decohere when the non-diagonal elements of the reduced density matrix are much smaller than the diagonal ones.

The decoherence time $t_{D}$ sets the scale after which we have a classical system-field configuration, and depends strongly on the properties of the environment. It satisfies

$$
1 \approx V \Delta^{2} \int_{0}^{t_{\mathrm{D}}} d s D\left(k_{0}, s\right)
$$

and corresponds to the time after which we are able to distinguish between two different field amplitudes, inside a given volume $V$.

To terms up to order $\lambda^{2}$ and one loop in the $\hbar$ expansion (we continue to work in units in which $\hbar=k_{B}=1$ ), the influence action due to the biquadratic interaction between system and environment has imaginary part

$$
\operatorname{Im} \delta S=-\int d^{4} x \int d^{4} y \Delta(x) N(x, y) \Delta(y),
$$

where $N(x, y)=\frac{1}{4} \lambda^{2} \operatorname{Re} G_{++}^{>2}(x, y)$ is the noise (diffusion) kernel and $G_{++}^{>}(x, y)$ is the thermal short-wavelength closed time-path correlator.

Explicit calculation shows that $D\left(k_{0}, t\right)$ takes the form

$$
D\left(k_{0}, t\right)=\int_{0}^{t} d s u(s, t) F\left(k_{0}, s, t\right)
$$

where

$$
u(s, t)=\left[u_{2}(s, t)-\frac{\dot{u}_{2}(t, t)}{\dot{u}_{1}(t, t)} u_{1}(s, t)\right]^{2},
$$

and $F\left(k_{0}, s, t\right)$ is built from the spatial Fourier transforms of the overlap of the diffusion kernel with the field profiles $\Phi(x) \Phi(y) \Phi(z)$.

In the integrand of $(21) u(s, t)$ is rapidly varying, driven by the unstable modes, and $F\left(k_{0}, s, t\right)$ is slowly varying. For long-wavelengths $k_{0} \ll \mu$ we have, approximately,

$$
F\left(k_{0}, s, t\right)=O\left(N\left(k_{0}=0 ; t-s\right)\right)
$$

whereby

$$
D\left(k_{0}, t\right) \approx F\left(k_{0}, 0, t\right) \int_{0}^{t} d s u(s, t)
$$

That is, the diffusion coefficient factorises into the environmental term $F$, relatively insensitive to both wavenumber and time, and the rapidly growing integral that measures the classical growth of the unstable system modes that are ordered in the transition.

To be specific, we restrict ourselves to the simplest case of an instantaneous quench from a temperature $T=$ $\mathcal{O}\left(T_{c}\right)>T_{C}$, for which

$$
u_{1}=\frac{\sinh \left[\omega_{0}(t-s)\right]}{\sinh \left(\omega_{0} t\right)}, u_{2}(s, t)=\frac{\sinh \left(\omega_{0} s\right)}{\sinh \left(\omega_{0} t\right)},
$$

where $\omega_{0}^{2}=\mu^{2}-k_{0}^{2} \approx \mu^{2}$. It follows that

$$
u(s, t)=\cosh ^{2}\left[\omega_{0}(t-s)\right],
$$

from whose end-point behaviour at $s=0$ of the integral (22) we find the even simpler result

$D\left(k_{0}, t\right) \sim \mu^{-1} F\left(k_{0}, 0, t\right) u(0, t) \sim\left(\lambda T_{\mathrm{c}} / 4 \pi \mu\right)^{2} \exp [2 \mu t]$,

assuming $\mu t_{D} \gg 1$.

For more general quenches growth is more complicated than simple exponential behaviour but a similar separation into fast and slow components applies.

We have omitted a large amount of complicated technical detail (see [6]), to give such a simple final result. This suggests that we could have reached the same conclusion more directly.

We now indicate how we can obtain the same results by demanding consistent histories of the $\phi$ field. 


\section{The Decoherence Functional}

The notion of consistent histories provides a parallel approach to classicality. Quantum evolution can be considered as a coherent superposition of fine-grained histories. If one defines the c-number field $\phi(x)$ as specifying a finegrained history, the quantum amplitude for that history is $\Psi[\phi] \sim e^{i S[\phi]}$ (we continue to work in units in which $\hbar=1)$.

In the quantum open system approach that we have adopted here, we are concerned with coarse-grained histories

$$
\Psi[\alpha]=\int \mathcal{D} \phi e^{i S[\phi]} \alpha[\phi]
$$

where $\alpha[\phi]$ is the filter function that defines the coarsegraining.

From this we define the decoherence function for two coarse-grained histories as

$\mathcal{D}\left[\alpha^{+}, \alpha^{-}\right]=\int \mathcal{D} \phi^{+} \mathcal{D} \phi^{-} e^{i\left(S\left[\phi^{+}\right]-S\left[\phi^{-}\right]\right)} \alpha^{+}\left[\phi^{+}\right] \alpha^{-}\left[\phi^{-}\right]$.

$\mathcal{D}\left[\alpha^{+}, \alpha^{-}\right]$does not factorise because the histories $\phi^{ \pm}$are not independent; they must assume identical values on a spacelike surface in the far future.

A necessary and sufficient condition for the validity of the sum rules of probability theory (i.e. no quantum interference terms) is [11]

$$
\operatorname{Re} \mathcal{D}\left[\alpha^{+}, \alpha^{-}\right] \approx 0,
$$

when $\alpha^{+} \neq \alpha^{-}$(although in most cases the stronger condition $\mathcal{D}\left[\alpha^{+}, \alpha^{-}\right] \approx 0$ holds [12]). Such histories are consistent [13].

For our particular application, we wish to consider as a single coarse-grained history all those fine-grained ones where the full field $\phi$ remains close to a prescribed classical field configuration $\phi_{\mathrm{cl}}$. The filter function takes the form

$$
\alpha_{\mathrm{cl}}[\phi]=\int \mathcal{D} J e^{i \int J\left(\phi-\phi_{\mathrm{cl}}\right)} \alpha_{\mathrm{cl}}[J] .
$$

In principle, we can examine general classical solutions for their consistency but, in practice, it is simplest to restrict ourselves to solutions of the form (13). In that case, we have made a de facto separation into long and short-wavelength modes whereby, in a saddle-point approximation over $J$,

$$
\mathcal{D}\left(\phi_{\mathrm{cl}}^{+}, \phi_{\mathrm{cl}}^{-}\right) \sim \exp \left\{i S_{C G}\left[\phi_{\mathrm{cl}}^{+}, \phi_{\mathrm{cl}}^{-}\right]\right\} \text {. }
$$

As a result,

$$
\left|\mathcal{D}\left(\phi_{\mathrm{cl}}^{+}, \phi_{\mathrm{cl}}^{-}\right)\right| \sim \exp \left\{-\operatorname{Im} \delta S\left[\phi_{\mathrm{cl}}^{+}, \phi_{\mathrm{cl}}^{-}\right]\right\}
$$

For the instantaneous quench of (14), using the late time behaviour $\phi_{\mathrm{cl}}^{ \pm} \sim e^{\mu s} \phi_{0}^{ \pm}, \operatorname{Im} \delta S\left[\phi_{\mathrm{cl}}^{+}, \phi_{\mathrm{cl}}^{-}\right]$takes the form

$$
\operatorname{Im} \delta S \sim \frac{V \Delta^{2}}{\mu^{2}} \int_{0}^{t} d s \int_{0}^{t} d s^{\prime} e^{2 \mu s} e^{2 \mu s^{\prime}} F\left(k_{0}, s, s^{\prime}\right) .
$$

From this viewpoint adjacent histories become consistent at the time $t_{D}$, for which

$$
1 \approx \int_{0}^{t_{D}} d t \operatorname{Im} \delta \mathrm{S}
$$

\section{The Decoherence Time}

We have used the same terminology for the time $t_{D}$ since, on inspection, (33) is identical to (19) in defining the onset of classical behaviour. As we noted, in practice the use of the decoherence functional looks to be less restrictive than the master equation, and we hope to show this elsewhere.

For the moment what is of interest is whether $t_{D}$, based on linearisation of the model, occurs before backreaction sets in, to invalidate this assumption. When all the details are taken onto account, whether from (14) or (23), $t_{D}$ satisfies

$$
1=\mathcal{O}\left(\frac{\lambda^{2} V T_{c}^{2}}{\mu^{3}} \Delta^{2}\right) \exp \left(4 \mu t_{D}\right)
$$

or, equivalently

$$
\exp \left(4 \mu t_{D}\right)=\mathcal{O}\left(\frac{\mu^{3}}{\lambda^{2} V T_{c}^{2} \Delta^{2}}\right)
$$

For the rapid quenches considered here, linearisation manifestly breaks down by the time $t^{*}$, for which $\left\langle\phi^{2}\right\rangle_{t^{*}} \sim \eta^{2}$, given by

$$
\exp \left(2 \mu t^{*}\right)=\mathcal{O}\left(\frac{\mu}{\lambda T_{\mathrm{c}}}\right)
$$

The exponential factor, as always, arises from the growth of the unstable long-wavelength modes. The factor $T_{\mathrm{c}}^{-1}$ comes from the $\operatorname{coth}(\beta \omega / 2)$ factor that encodes the initial Boltzmann distribution at temperature $T \gtrsim T_{\mathrm{c}}$.

Our conservative choice is that the volume factor $V$ is $\mathcal{O}\left(\mu^{-3}\right)$ since $\mu^{-1}$ (the Compton wavelength) is the smallest scale at which we need to look. With this choice it follows that

$$
\left.\exp 2\left(t^{*}-t_{D}\right)=\mathcal{O}\left(\frac{|\Delta|}{\mu^{2}}\right)\right)=\mathcal{O}(\bar{\phi} \delta),
$$

where $\bar{\phi}=\left(\phi_{<}^{+}+\phi_{<}^{-}\right) / 2 \mu$, and $\delta=\left|\phi_{<}^{+}-\phi_{<}^{-}\right| / 2 \mu$. Within the volume $V$ we do not discriminate between field amplitudes which differ by $\mathcal{O}(\mu)$, and therefore take $\delta=\mathcal{O}(1)$. For $\bar{\phi}$ we note that, if $t_{D}$ were to equal $t^{*}$, then $\bar{\phi}^{2}=$ $\mathcal{O}(1 / \lambda) \gg 1$, and in general $\bar{\phi}>1$. As a result, if there are no large numerical factors, we have

$$
t_{D}<t^{*}
$$

and the density matrix has become diagonal before the transition is complete. Detailed calculation shows [6] that there are no large factors [14].

We already see a significant difference between the behaviour for the case of a biquadratic interaction with an environment given in (19) and the more familiar linear interaction usually adopted in quantum mechanics. This latter would have replaced $\Delta / \mu^{2}$ just by $\delta$, incapable of inducing decoherence before the transition is complete.

We note that, once the interaction strength is sufficiently weak for classical behaviour to appear before the transition 
is complete, this persists, however weak the coupling becomes. It remains the case that, the weaker the coupling, the longer it takes for the environment to decohere the system but, at the same time, the longer it takes for the transition to be completed, and the ordering (38) remains the same. This is equally true for more general quenches provided the system remains approximately Gaussian until the transition is complete.

\section{Extensions of the Model}

Finally, it has to be said that taking only the short wavelength modes of the field as a one-loop system environment is not a robust approximation. We should sum over hard thermal loops in the $\phi$-propagators. To be in proper control of the diffusion we need an environment that interacts with the system, without the system having a strong impact on the environment. We are helped in that, in the early universe, the order parameter field $\phi$ will interact with any field $\chi$ for which there is no selection rule. Again, it is the biquadratic interactions that are the most important.

The most simple additional environment is one of a large number $N \gg 1$ of weakly coupled scalar fields $\chi_{a}$, for which the action (1) is extended to

$$
S[\phi, \chi]=S[\phi]+S[\chi]+S_{\text {int }}[\phi, \chi],
$$

where $S[\phi]$ is as before, and

$$
\begin{aligned}
& S\left[\chi_{\mathrm{a}}\right]=\sum_{\mathrm{a}=1}^{N} \int d^{4} x\left\{\frac{1}{2} \partial_{\mu} \chi_{\mathrm{a}} \partial^{\mu} \chi_{\mathrm{a}}-\frac{1}{2} m_{\mathrm{a}}^{2} \chi_{\mathrm{a}}^{2}\right\}, \\
& S_{\mathrm{int}}[\phi, \chi]=-\sum_{\mathrm{a}=1}^{N} \frac{g_{\mathrm{a}}}{8} \int d^{4} x \phi^{2}(x) \chi_{\mathrm{a}}^{2}(x),
\end{aligned}
$$

where $m_{\mathrm{a}}^{2}>0$. For simplicity we take weak couplings $\lambda \simeq g_{\mathrm{a}}$ and comparable masses $m_{\mathrm{a}} \simeq \mu$. The effect of a large number of weakly interacting environmental fields is twofold. Firstly, the $\chi_{a}$ fields reduce the critical temperature $T_{\mathrm{c}}$ and, in order that $T_{\mathrm{c}}^{2}=\frac{2 \mu^{2}}{\lambda+\sum g_{\mathrm{a}}} \gg \mu^{2}$, we must take $\lambda+\sum g_{\mathrm{a}} \ll 1$. Secondly, the single $\chi$-loop contribution to the diffusion coefficient is the dominant $\chi$-field effect if, for order of magnitude estimates, we take identical $g_{\mathrm{a}}=\bar{g} / \sqrt{N}$, whereby $1 \gg 1 / \sqrt{N} \gg \bar{g} \simeq \lambda$. With this choice the effect of the $\phi$-field on the $\chi_{\mathrm{a}}$ thermal masses is, relatively, $O(1 / \sqrt{N})$ and can be ignored. We stress that this is not a Hartree or large-N approximation of the type that, to date, has been the main way to proceed $[15,16,17]$ for a closed system.

Provided the change in temperature is not too slow the exponential instabilities of the $\phi$-field grow so fast that the field has populated the degenerate vacua well before the temperature has dropped to zero. Since the temperature $T_{c}$ has no particular significance for the environment field, for these early times we can keep the temperature of the environment fixed at $T_{\chi}=\mathcal{O}\left(T_{\mathrm{c}}\right)$ (our calculations are only at the level of orders of magnitude). As before, we split the field as $\phi=\phi_{<}+\phi_{>}$. The $\chi$-fields give an additional oneloop contribution to $D\left(k_{0}, t\right)$ with the same $u(s)$ but a $G_{++}$ constructed from (all the modes of) the $\chi$-field. The separation of the diffusion coefficient due to $\chi$ into fast and slow factors proceeds as before to give a term that is identical to (25) (or (32)) but for its $\bar{g}^{2}$ prefactor.

Diffusion effects are additive at the one-loop level, and the final effect is to replace $\lambda^{2}$ in (34) by $\lambda^{2}+\bar{g}^{2}>\lambda^{2}$, while leaving (36) unchanged. Although the relationship between $T_{\mathrm{c}}$ and $\lambda$ has been uncoupled by the presence of the $\chi_{\mathrm{a}}$, the relationship (37) persists, with an enhanced right hand side, requiring that (38) is even better satisfied.

Given that the effect of further environmental fields is to increase the diffusion coefficient and speed up the onset of classical behaviour, additional fields interacting with the $\phi$ field seem superfluous. However, the symmetries of the universe seem to be local (gauge symmetries), rather than global, and we should take gauge fields into account. We conclude with some observations from our work in progress [18] with local symmetry breaking.

Local symmetry breaking is not possible for our real $\phi$ field but, as a first step [5], it is not difficult to extend our model to that of a complex $\phi$-field. At the level of $O(2)$ global interactions with external fields and with its own short-wavelength modes, things are largely as before. Local $U(1)$ symmetry breaking is most easily accommodated by taking the $\phi$-field to interact with other charged fields $\chi$ through the local $U(1)$ action

$$
S\left[\phi, A_{\mu}, \chi\right]=S\left[\phi, A_{\mu}\right]+S_{\chi}\left[A_{\mu}, \chi\right],
$$

in which $S\left[\phi, A_{\mu}\right]=$

$$
\int d^{4} x\left\{\left(D_{\mu} \phi\right)^{*} D^{\mu} \phi+\mu^{2} \phi^{*} \phi-\frac{\lambda}{4}\left(\phi^{*} \phi\right)^{2}-\frac{1}{4} F^{\mu \nu} F_{\mu \nu}\right\},
$$

and

$$
S\left[A_{\mu}, \chi\right]=\int d^{4} x\left\{\left(D_{\mu} \chi\right)^{*} D^{\mu} \chi+m^{2} \chi^{*} \chi\right\} .
$$

For simplicity we have taken a single $\chi$ field. The theory (41) shows a phase transition at temperature $T_{c}$, and we assume couplings are such as to make this transition continuous. At the level of one loop the additional term to the diffusion function has derivative couplings. Having made a gauge choice, these give rise to explicit momenta factors $k_{\mu}$ in the generalisation of $F$. Unlike the contributions to $D$ that we have seen so far, which are largely insensitive to the momentum scale $k_{0}$, these contributions are strongly damped at large wavelength. In consequence, they barely enhance the onset of classical behaviour but, given that the effect of the other environmental modes is to enforce classical behaviour so quickly, it hardly matters.

\section{Conclusion}

The previous paragraph says it all. For fast quenches weakly coupled environments make a scalar order parameter field decohere before the transition is complete, under very general assumptions. An essential ingredient for rapid decoherence is nonlinear coupling to the environment, inevitable 
when that environment contains the short-wavelength modes of the order parameter field. Had we only considered linear coupling to the environment, as in [19], for example (but an assumption that is ubiquitous in quantum mechanical models, from Brownian motion onwards) decoherence would not have happened before the transition was complete, and we would not know how to proceed, although classical correlations would have occurred.

\section{Acknowledgments}

We thank Diego Mazzitelli for his collaboration in this work. F.C.L. was supported by Universidad de Buenos Aires, CONICET (Argentina), Fundación Antorchas and ANPCyT. R.J.R. was supported in part by the COSLAB programme of the European Science Foundation.

\section{References}

[1] E. Kolb and M. Turner, The Early Universe (Addison Wesley, Reading, Mass., 1990).

[2] A. Guth and S.Y. Pi, Phys. Rev. D 32, 1899 (1991).

[3] F.C. Lombardo, F.D. Mazzitelli, and D. Monteoliva, Phys. Rev. D 62, 045016 (2000).

[4] F.C. Lombardo, F.D. Mazzitelli, and R.J. Rivers, Phys. Lett. B 523, 317 (2001).

[5] R.J. Rivers, F.C. Lombardo, and F.D. Mazzitelli, Phys. Lett. B 539, 1 (2002).

[6] R.J. Rivers, F.C. Lombardo, and F.D. Mazzitelli, Nucl. Phys. B 672, 462 (2003).
[7] F.C. Lombardo, Influence functional approach to decoherence during Inflation, these proceedings.

[8] F.C. Lombardo and F.D. Mazzitelli, Phys. Rev. D 53 (1996) 2001.

[9] R. Feynman and F. Vernon, Ann. Phys. (N.Y.) 24, 118 (1963).

[10] T.W.B. Kibble, Phys. Rep. 67, 183 (1980).

[11] R.B. Griffiths, J. Stat. Phys. 36, 219 (1984).

[12] R. Omnès, J. Stat. Phys. 53, 893 (1988); Ann. Phys. 201 (1990) 354; Rev. Mod. Phys. 64, 339 (1992).

[13] M. Gell-Mann and J.B. Hartle, Phys. Rev. D 47, 3345 (1993); J.J. Halliwell, Phys. Rev. D 60, 105031 (1999).

[14] In evaluating $F$ we perform the loop diagram using the full propagator rather than just its short wavelength modes. Since the $T_{\mathrm{c}}$ behaviour comes entirely from the short wavelength part of the integral, this is justified.

[15] D. Boyanovsky, H.J. de Vega, and R. Holman, Phys. Rev. D 49, 2769 (1994); D. Boyanovsky, H.J. de Vega, R. Holman, D.-S. Lee, and A. Singh, Phys. Rev. D 51, 4419 (1995); S.A. Ramsey and B.L. Hu, Phys. Rev. D 56, 661 (1997).

[16] F. Cooper, S. Habib, Y. Kluger, and E. Mottola, Phys. Rev. D 55, 6471 (1997).

[17] G.J. Stephens, E.A. Calzetta, B.L. Hu, and S.A. Ramsey, Phys. Rev. D 59, 045009 (1999).

[18] N.G. Busca, F.C. Lombardo, F.D. Mazzitelli, and R.J. Rivers, work in progress.

[19] S.P. Kim and C.H. Lee, Phys. Rev. D 65, 045013 (2002). 